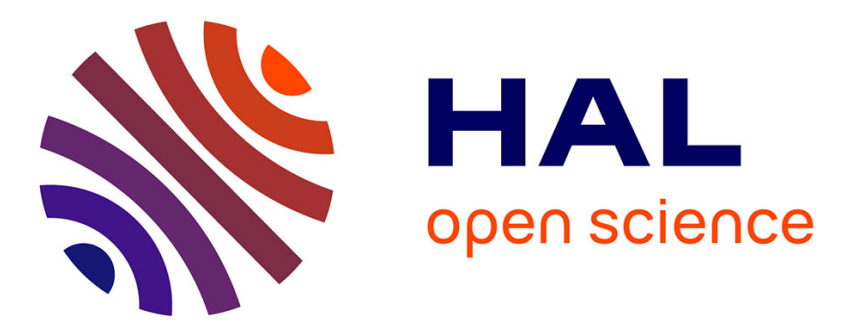

\title{
Simultaneous observer based sensor diagnosis and speed estimation of Unmanned Aerial Vehicle
}

\author{
Hugues Rafaralahy, Edouard Richard, Mohamed Boutayeb, Michel \\ Zasadzinski
}

\section{- To cite this version:}

Hugues Rafaralahy, Edouard Richard, Mohamed Boutayeb, Michel Zasadzinski. Simultaneous observer based sensor diagnosis and speed estimation of Unmanned Aerial Vehicle. 47th IEEE Conference on Decision and Control, CDC'08, Dec 2008, Cancun, Mexico. pp.CDROM. hal-00344913

\section{HAL Id: hal-00344913 \\ https://hal.science/hal-00344913}

Submitted on 6 Dec 2008

HAL is a multi-disciplinary open access archive for the deposit and dissemination of scientific research documents, whether they are published or not. The documents may come from teaching and research institutions in France or abroad, or from public or private research centers.
L'archive ouverte pluridisciplinaire HAL, est destinée au dépôt et à la diffusion de documents scientifiques de niveau recherche, publiés ou non, émanant des établissements d'enseignement et de recherche français ou étrangers, des laboratoires publics ou privés. 


\title{
Simultaneous observer based sensor diagnosis and speed estimation of Unmanned Aerial Vehicle
}

\author{
H. Rafaralahy*, E. Richard $\dagger$ M. Boutayeb* and M. Zasadzinski*
}

\begin{abstract}
In this contribution we investigate the problem of simultaneous observer based sensor diagnosis and speed estimation of Unmanned Aerial Vehicle (UAV). The main features lie in the use of a useful bank of reduced order observers to detect and isolate faulty sensors and in the same time to provide unbiased speed estimation of UAV from accelerometers. From a structural analysis, we provide all trajectories for which faults would be detected and estimated. The theoretical result is summarized into the stability analysis, established through the Lyapunov approach and Barbalat lemma. The existence condition is expressed in terms of physical variables of the UAV. Performances and easiness of implementation of the proposed technique are shown through a quadrotor UAV.

Index Terms - Unmanned Aerial Vehicle (UAV), Quadrotor, Speed estimation, Time-varying observer, Stability analysis, Fault diagnosis, Sensor fault estimation.
\end{abstract}

\section{Introduction}

UAV and more particularly mini-UAV must be as light as possible for technological reasons but also for low cost and low energy consumption. In fact, the weight of the drone can reduce its performances considerably. It is therefore rather natural to use a state estimator to replace possibly one or several sensors. Observers are usually used to estimate the non measured state components for output feedback control or to generate residual signals for fault diagnosis. During the last decade, tremendous research activities focused on structural, modelling and control design for UAV (see for example [1], [13], [15]). However, very few results were established for the state estimation. Among the recent works on this subject, we mention the work of [1], the authors propose a velocities estimator for a tracking control of an under-actuated quadrotor UAV using only linear and angular positions. In [2] and [3], sliding-mode observers based control are proposed to estimate the effect of external perturbations using measurement of positions and yaw angle. Observer-based control for visual servo control of UAV has been proposed for example in [4] using image provided by a camera for the estimation of the velocity. Extended Kalman Filter is used in [8] to estimate the state of a moving object detected by a UAV. The disadvantage of this method is its only local convergence. All the results cited above are obtained using linear and angular positions.

In [9], the authors propose a nice technique to estimate the speed of a quadrotor UAV from acceleration measurements, provided by Inertial Measurement Units. The approach is based on an adaptive observer technique using cascade nonlinear filters that leads, unfortunately, to a high order observer and therefore, the computational requirements increase considerably for on-line control purpose. More precisely, the observer is composed with two matrix differential equations in cascade with two nonlinear filters. In [12] a simple time-varying reduced-order observer has been presented to estimate the linear velocity of an UAV using full or partial acceleration measurements.

For the use of observers in fault diagnosis, we can mention for example [5], in which two cascaded sliding mode observers are proposed first to estimate the disturbances (effect of wind) and second to reconstruct actuators faults. In [6], a linear input decoupled functional observer is proposed to estimate

\footnotetext{
*The authors are with Centre de Recherche en Automatique de Nancy (CRAN-UMR 7039) Nancy-University - CNRS, 186 rue de Lorraine, 54400 Longwy, France, E-mail : Hugues.Rafaralahy@iut-longwy.uhp-nancy.fr

${ }^{\dagger}$ IUT de Longwy, Nancy-University, 186 rue de Lorraine, 54400 Longwy, France, E-mail : Edouard.Richard@iutlongwy.uhp-nancy.fr
} 
actuators failures of UAV. The proposed method is based on the linearization of the model around an operating point. A variable structure observer is performed in [7] for actuator bias reconstruction of UAV without using a linearization. In [18], Piercy used detection filter residuals for the detection and identification of sensor failures for discrete time linear systems. The simultaneous multiplicative actuator and sensor fault estimation using linear observer and adaptive rules for the fault estimation is related in [11]. The analytical redundancy approach has been applied to the sensor failures detection and identification for F8 aircraft and the robot navigation problem in [14] and [16] respectivelly.

In [19] and [20] the authors proposed simultaneous state and sensor fault estimation for nonlinear and bilinear systems. Motivated by the previous works, our approach is based on simultaneous state and sensor fault estimation through a straightforward bank of reduced-order time-varying observers without using the singular state-space framework. Asymptotic stability conditions that are reduced to simple and checkable one are provided.

\section{UAV modelling}

Several works deal with the quadrotor modelling (see for example [13] and [15]). In this section we recall a sketch of modelling as well as the notations are introduced. In order to model the four-rotor rotorcraft dynamics two frames are defined i.e. $\mathcal{R}_{i}\left(O, \vec{E}_{1}, \vec{E}_{2}, \vec{E}_{3}\right)$ is an inertial frame attached to the earth and $\mathcal{R}_{b}\left(G, \vec{e}_{1}, \vec{e}_{2}, \vec{e}_{3}\right)$ is a body fixed frame attached to its center of mass (see fig. 1).

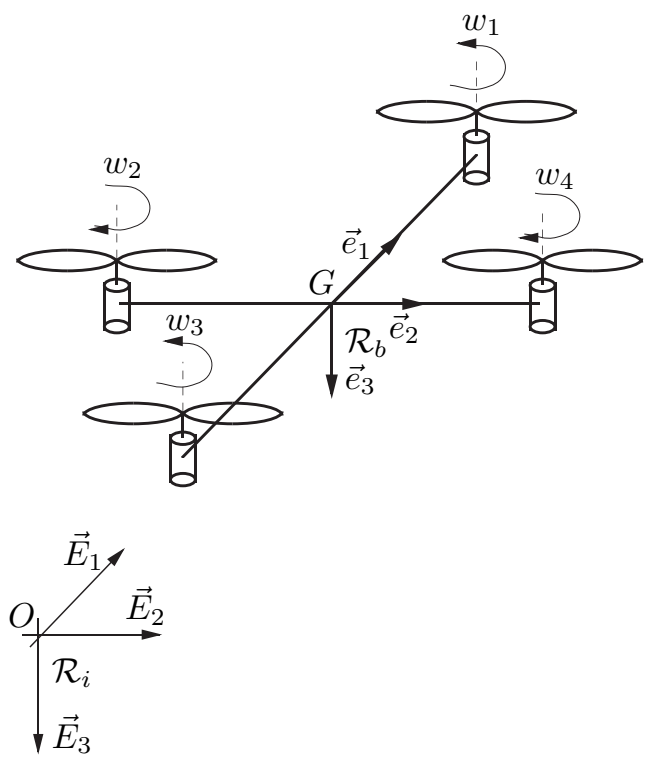

Figure 1: Quadrotor model

In order to obtain the body fixed frame $\mathcal{R}_{b}$ from the inertial one $\mathcal{R}_{i}$ three rotations are used: the Cardan angles [17]. The first one sequentially rotates about $\vec{E}_{3}$ axis ( $\Psi$ yaw) resulting in an intermediate second frame $\left(\vec{E}_{1}^{\prime}, \vec{E}_{2}^{\prime}, \vec{E}_{3}^{\prime}=\vec{E}_{3}\right)$, then about $\vec{E}_{2}^{\prime}$ axis $(\theta$ pitch) resulting an intermediate third frame $\left(\vec{E}_{1}^{\prime \prime}=\vec{e}_{1}, \vec{E}_{2}^{\prime \prime}=\vec{E}_{2}^{\prime}, \vec{E}_{3}^{\prime \prime}\right)$ and finally a rotation around $\vec{e}_{1}$ ( $\Phi$ roll) leads to the body fixed frame (see fig. 2).

Now let $\underline{X}_{\mathcal{R}_{i}}$ and $\underline{X}_{\mathcal{R}_{b}}$ be the coordinates of a three dimensional vector $\underline{X}$ projected onto the inertial frame $\mathcal{R}_{i}$ and the body frame $\mathcal{R}_{b}$ respectively and $R$ be the matrix attitude composed by the three rotations $\Phi, \theta$ and $\psi$. Then $\underline{X}_{\mathcal{R}_{i}}$ and $\underline{X}_{\mathcal{R}_{b}}$ are related by the following relation

$$
\underline{X}_{\mathcal{R}_{i}}=R \underline{X}_{\mathcal{R}_{b}} \quad \text { or } \quad \underline{X}_{\mathcal{R}_{b}}=R^{T} \underline{X}_{\mathcal{R}_{i}}
$$

with

$$
R=\left(\begin{array}{ccc}
c_{\theta} c_{\Psi} & s_{\Phi} s_{\theta} c_{\Psi}-c_{\Phi} s_{\Psi} & c_{\Phi} s_{\theta} c_{\Psi}+s_{\Phi} s_{\Psi} \\
c_{\theta} s_{\Psi} & s_{\Phi} s_{\theta} s_{\Psi}+c_{\Phi} c_{\Psi} & c_{\Phi} s_{\theta} s_{\Psi}-s_{\Phi} c_{\Psi} \\
-s_{\theta} & s_{\Phi} c_{\theta} & c_{\Phi} c_{\theta}
\end{array}\right)
$$


where $c_{.}=\cos ($.$) and s .=\sin ($.$) . Then, from the angles choice, the vector of angular velocity \vec{\omega}$ can be written as

$$
\vec{\omega}=\dot{\Psi} \vec{E}_{3}+\dot{\theta} \vec{E}_{2}^{\prime}+\dot{\Phi} \vec{e}_{1}
$$
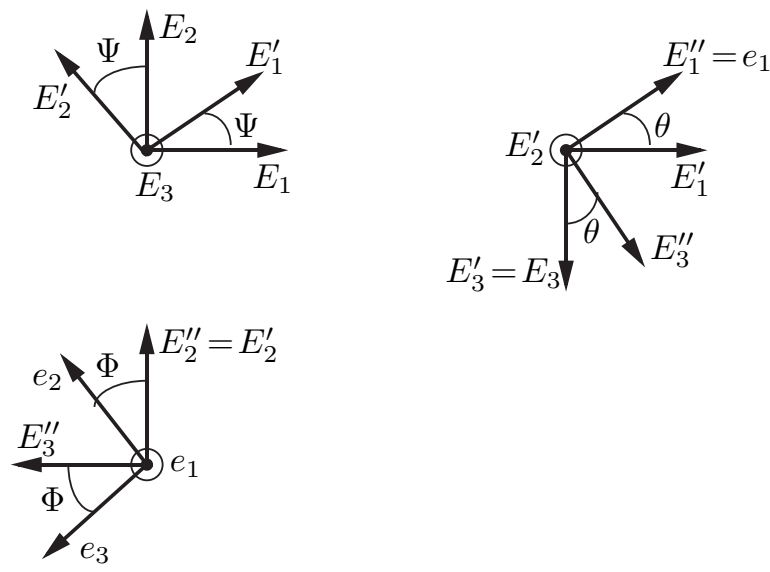

Figure 2: Cardan angles

Thanks to relation (2) and by projecting all vectors onto the body frame, the time derivative of the Cardan angles are related to the angular velocity $\vec{\omega}=(p, q, r)^{T}$ by the following relation

$$
\left\{\begin{aligned}
\dot{\Phi} & =p+q \tan \theta \sin \Phi+r \tan \theta \cos \Phi \\
\dot{\theta} & =q \cos \Phi-r \sin \Phi \\
\dot{\psi} & =q \frac{\sin \Phi}{\cos \theta}+r \frac{\cos \Phi}{\cos \theta}
\end{aligned}\right.
$$

The UAV dynamical model can be deduced from the rotation dynamic Newton-Euler law (4) and the translation dynamic Newton-Euler law (5)

$$
\begin{gathered}
\left(\frac{\mathrm{d} \mathbf{I} \vec{\omega}}{\mathrm{d} t}\right)_{\mathcal{R}_{b}}+\vec{\omega} \wedge(\mathbf{I} \vec{\omega})=\overrightarrow{\mathcal{M}} \\
m\left(\frac{\mathrm{d} \vec{v}_{G}}{\mathrm{~d} t}\right)_{\mathcal{R}_{b}}+\vec{\omega} \wedge\left(m \vec{v}_{G}\right)=\vec{F}
\end{gathered}
$$

where $\mathbf{I}$ is the inertia matrix and $\vec{\omega}=(p, q, r)^{T}$ is the angular velocity both expressed in the body fixed frame. $\overrightarrow{\mathcal{M}}$ represents the torque derived from the differential rotors thrusts. $m$ is the vehicle mass, $\vec{v}_{G}=(u, v, w)^{T}$ is the center of mass velocity with respect to the inertial frame $\mathcal{R}_{i}$ and projected onto the body fixed frame. $\vec{F}$ is the sum of the four rotor thrust $\vec{T}=-T \vec{e}_{3}$ and the gravity $\vec{P}=m g \vec{E}_{3}$. In order to express the gravity with respect to the body fixed frame, the attitude matrix $R$ must be used (eq. (1)).

From the rotation dynamic Newton-Euler law (4) the dynamics of the angular velocity projected onto $\mathcal{R}_{b}$ is given by

$$
\left\{\begin{array}{l}
\dot{p}=-\frac{I_{z z}-I_{y y}}{I_{x x}} q r+\frac{\tau_{\Phi}}{I_{x x}} \\
\dot{q}=-\frac{I_{x x}-I_{z z}}{I_{y y}} p r+\frac{\tau_{\theta}}{I_{y y}} \\
\dot{r}=-\frac{I_{y y}-I_{x x}}{I_{z z}} p q+\frac{\tau_{\psi}}{I_{z z}}
\end{array}\right.
$$

where $I_{x x}, I_{y y}$ and $I_{z z}$ are the inertia matrix terms expressed in the principal inertia axis, $\tau_{\Phi}, \tau_{\theta}$ and $\tau_{\psi}$ represent the control torques due to the differential rotors thrusts. Using the center of mass dynamics equation (5) and the attitude matrix (1), the translation dynamics with respect to the body frame is 


$$
\left\{\begin{array}{l}
\dot{u}=-q w+r v-g \sin \theta \\
\dot{v}=-r u+p w+g \sin \Phi \cos \theta \\
\dot{w}=-p v+q u+g \cos \Phi \cos \theta-\frac{T}{m}
\end{array}\right.
$$

where $T$ is the total thrust which represents a control input. The dynamical model of the four-rotor rotorcraft is then given by equations (3), (6) and (7).

\section{Problem statement}

Since the UAV is equiped only with an Inertial Measurement Unit it is assumed that the measured variables are the Cardan angles $\eta=(\Phi, \theta, \psi)^{T}$, the angular velocity $\vec{\omega}$ and the acceleration of the center of mass $(\dot{u}, \dot{v}, \dot{w})^{T}$ given by the sensors embedded in the four-rotor rotorcraft. The states of equations (6) and (3) are then measured.

The aim of this contribution is the synthesis of a bank of observers or state and sensor fault observer and residual generator (SFORG) whose tasks consist

- in estimating the linear velocity of the quadrotor,

- in generating residuals to detect the accelerometers faults,

- and in locating and reconstructing these faults.

Each SFORG is based on the model (7) (thus of dimension three) and uses two measurements of acceleration for the speed estimation and the residual generation. If the sensors measuring these two accelerations are free fault, then the residues provided by this SFORG are null and the state estimation errors is asymptotically stable. Then the third acceleration measurement is used to reconstruct the fault sensor. In the case of one or two sensors are faulty, the residues of this SFORG are different from zero and the estimation errors are not asymptotically stable.

Let $x=(u, v, w)^{T}$ be the linear velocity to be estimated. We consider the model of system (7). Taking into account the measured variables, the system (7) can be rewritten as

$$
\begin{aligned}
\dot{x}(t) & =A(t) x(t)+b(t) \\
y(t) & =\dot{x}(t)+f(t)
\end{aligned}
$$

with

$$
A(t)=\left(\begin{array}{ccc}
0 & r & -q \\
-r & 0 & p \\
q & -p & 0
\end{array}\right)
$$

and

$$
b(t)=\left(\begin{array}{c}
-g \sin \theta \\
g \sin \Phi \cos \theta \\
g \cos \Phi \cos \theta-\frac{T}{m}
\end{array}\right)
$$

where $f(t)=\left[\begin{array}{lll}f_{1}(t) & f_{2}(t) & f_{3}(t)\end{array}\right]^{T}$ is the time-varying fault vector. We assume that at every time $t$, only one sensor is faulty. In this paper, we consider additive time varying faults occuring abruptly or incipiently. The class of considered faults includes bias, drifts and possibly the variations of the sensor gain (see [19] and [21]).

\section{Observer design}

The proposed state and sensor fault observer and residual generator (SFORG) has the following form for $i=1,2,3$

$$
\left(\mathcal{O}_{i}\right)\left\{\begin{aligned}
\dot{\hat{x}}^{i}(t) & =N_{i}(t) \hat{x}^{i}(t)+M_{i}(t) b(t)+K_{i}(t) Y^{i}(t) \\
\hat{f}_{i}(t) & =\bar{Y}^{i}(t)-\bar{C}_{i}(t) \dot{\hat{x}}^{i}(t) \\
r_{i}(t) & =Q_{i}(t) \dot{\hat{x}}^{i}(t)+P_{i}(t) Y^{i}(t)
\end{aligned}\right.
$$


with

$$
\begin{aligned}
& Y^{i}(t)=C_{i} y(t)=C_{i} \dot{x}+\bar{f}^{i}(t) \\
& \bar{Y}^{i}(t)=\bar{C}_{i} y(t)=\bar{C}_{i} \dot{x}+f_{i}(t)
\end{aligned}
$$

and $\bar{f}^{1}(t)=\left[\begin{array}{ll}f_{2}(t) & f_{3}(t)\end{array}\right]^{T}, \bar{f}^{2}(t)=\left[\begin{array}{ll}f_{1}(t) & f_{3}(t)\end{array}\right]^{T}$ and $\bar{f}^{3}(t)=\left[\begin{array}{ll}f_{1}(t) & f_{2}(t)\end{array}\right]^{T}$

$$
\begin{aligned}
& C_{1}=\left[\begin{array}{lll}
0 & 1 & 0 \\
0 & 0 & 1
\end{array}\right] \bar{C}_{1}=\left[\begin{array}{lll}
1 & 0 & 0
\end{array}\right], \\
& C_{2}=\left[\begin{array}{lll}
1 & 0 & 0 \\
0 & 0 & 1
\end{array}\right] \bar{C}_{2}=\left[\begin{array}{lll}
0 & 1 & 0
\end{array}\right], \\
& C_{3}=\left[\begin{array}{lll}
1 & 0 & 0 \\
0 & 1 & 0
\end{array}\right] \bar{C}_{3}=\left[\begin{array}{lll}
0 & 0 & 1
\end{array}\right],
\end{aligned}
$$

where $\hat{x}^{i}(t), \hat{f}_{i}(t)$ are the estimates of the state $x(t)$ and component of the sensor fault vector $f_{i}(t)$ respectively and $r_{i}(t)$ is the $i^{\text {th }}$ residual.

Assumption 1. $\vec{\omega}(t), \dot{\vec{\omega}}(t)$, and $\ddot{\vec{\omega}}(t)$ are bounded.

Remark 1. It is easy to see that if assumption (1) is fullfilled then matrices $A, \dot{A}$ and $\ddot{A}$ are bounded.

Assumption 2. At least one component of the vector $\vec{\omega} \wedge \dot{\vec{\omega}}$ does not go to zero at infinity .

Remark 2. Let $\vec{\omega}=\omega \vec{u}$ with $\|\vec{u}\|=1$. Then $\dot{\vec{u}} \cdot \vec{u}=0$ and $\dot{\vec{\omega}} \wedge \vec{\omega}=\omega^{2} \vec{u} \wedge \dot{\vec{u}}$. If follows that the assumption 2 means that $\dot{\vec{u}} \neq 0$, that is the direction of $\vec{\omega}$ is varying.

Lemma 1. Assume that the previous assumptions hold and the matrices $N_{i}(t), M_{i}(t), K_{i}(t), Q_{i}$ and $P_{i}$ are chosen as

$$
\begin{aligned}
K_{i}(t) & =L_{i}+\gamma_{i} A^{T}(t) C_{i}^{T} \\
N_{i}(t) & =A(t)-K_{i}(t) C_{i} A(t) \\
M_{i}(t) & =I-K_{i}(t) C_{i} \\
Q_{i} & =-\beta_{i} C_{i} \\
P_{i} & =\beta_{i} I
\end{aligned}
$$

with

$$
\begin{aligned}
& L_{1}(t)=\left(\begin{array}{cc}
-q / p & -r / p \\
1 & 0 \\
0 & 1
\end{array}\right), \\
& L_{2}(t)=\left(\begin{array}{cc}
1 & 0 \\
-p / q & -r / q \\
0 & 1
\end{array}\right), \\
& L_{3}(t)=\left(\begin{array}{cc}
1 & 0 \\
0 & 1 \\
-p / r & -q / r
\end{array}\right)
\end{aligned}
$$

where $\gamma_{i}$ and $\beta_{i}$ are strictly positive tuning parameters then the following statements hold. 
1. If $\bar{f}^{i}(t) \equiv 0$ then the dynamics of the observation error $\epsilon^{i}=x-\hat{x}^{i}$ is asymptoticaly stable at the origin and $r_{i}(t) \rightarrow 0$ and $\hat{f}_{i}(t) \rightarrow f_{i}(t)$ as $t \rightarrow \infty$.

2. If $\bar{f}^{i}(t) \neq 0$ i.e. $f_{j}(t) \neq 0$ for $j \neq i$ and $f_{j}(t) \neq \dot{\sigma}_{j}$ with $\sigma$ solution of $\dot{\sigma}(t)=A(t) \sigma(t)$ then $r_{i}(t) \neq 0$ and $r_{i}(t) \nrightarrow 0$ ( $\sigma_{j}$ is the $j^{\text {th }}$ component of $\left.\sigma\right)$. 
Proof. Using equations (8) and (9) the observation error $\epsilon^{i}=x-\hat{x}^{i}$ dynamics, the $i^{\text {th }}$ residual $r_{i}(t)$ and the sensor fault estimation error $\epsilon_{f}^{i}(t)=f_{i}(t)-\hat{f}_{i}(t)$ can be written as

$$
\begin{aligned}
\dot{\epsilon}^{i}(t) & =N_{i} \epsilon^{i}(t)+\left(A-N_{i}-K_{i} C_{i} A\right) x(t) \\
& +\left(I-M_{i}-K_{i} C_{i}\right) b(t)-\left(L_{i}+\gamma_{i} A^{T} C_{i}^{T}\right) \bar{f}^{i}(t) \\
r_{i}(t) & =P_{i}\left(C_{i} \dot{\epsilon}^{i}(t)+\bar{f}^{i}(t)\right) \\
\epsilon_{f}^{i}(t) & =-\bar{C}_{i} \dot{\epsilon}^{i}(t)
\end{aligned}
$$

It is easy to see that the unbiasedness conditions

$$
\begin{aligned}
A-N_{i}-K_{i} C_{i} A & =0 \\
I-M_{i}-K_{i} C_{i} & =0
\end{aligned}
$$

are satisfied if matrices $N_{i}(t), M_{i}(t)$ and $K_{i}(t)$ are chosen as in (10).

Using the expression of $K_{i}(t)$, one obtains

$$
N_{i}(t)=A(t)-L_{i}(t) C_{i} A(t)-\gamma_{i} A^{T}(t) C_{i}^{T} C_{i} A(t)
$$

Now, choosing $L_{i}$ as in (11) yields $A(t)-L_{i}(t) C_{i} A(t)=0$ and finally the observation error becomes

$$
\dot{\epsilon}^{i}(t)=-\gamma_{i} A^{T}(t) C_{i}^{T} C_{i} A(t) \epsilon^{i}(t)-\left(L_{i}+\gamma_{i} A^{T} C_{i}^{T}\right) \bar{f}^{i}(t)
$$

In what follows, the proof is made in two steps. In the first step, we show that if $\bar{f}^{i}(t) \equiv 0$ then the observation error $\epsilon^{i}=x-\hat{x}^{i}$ dynamics is asymptoticaly stable, the $i^{\text {th }}$ residual $r_{i}(t)$ and the sensor fault estimation error $\epsilon_{f}^{i}(t)$ converge to 0 .

First consider the case $\bar{f}^{i}(t) \equiv 0$. Then the observation error becomes

$$
\dot{\epsilon}^{i}(t)=-\gamma_{i} A^{T}(t) C_{i}^{T} C_{i} A(t) \epsilon^{i}(t)
$$

Consider the following Lyapunov function candidate $V\left(\epsilon^{i}\right)=\epsilon^{i T} \epsilon^{i}$. The time derivative of $V$ along the observation error dynamics (16) leads to

$$
\dot{V}=-2 \gamma_{i}\left\|C_{i} A \epsilon^{i}\right\|^{2} \leq 0
$$

Since $V\left(\epsilon^{i}(t)\right)$ is positive definite and non-increasing, $V\left(\epsilon^{i}(t)\right)$ converges asymptotically to some limit value i.e. $V\left(\epsilon^{i}(t)\right) \rightarrow \ell$ as $t \rightarrow \infty$ where $\ell$ is finite. Then $\epsilon^{i}(t)$ is bounded.

Now, we use Barbalat's lemma in order to prove that $\dot{V}(t) \rightarrow 0$. The time derivative of $\dot{V}$ along equation (16) gives

$$
\begin{aligned}
\ddot{V}\left(\epsilon^{i}(t)\right) & =4 \gamma_{i}^{2} \epsilon^{i T} A^{T} C_{i}^{T} C_{i} A A^{T} C_{i}^{T} C_{i} A \epsilon^{i} \\
& -4 \gamma_{i} \epsilon^{i T} \dot{A}^{T} C_{i}^{T} C_{i} A \epsilon^{i} .
\end{aligned}
$$

Since $\epsilon^{i}(t), A(t), \dot{A}(t)$ are bounded then $\ddot{V}$ is bounded, which implies that $\dot{V}\left(\epsilon^{i}(t)\right)$ is uniformly continuous. Now using Barbalat's lemma it follows that $\dot{V}\left(\epsilon^{i}(t)\right) \rightarrow 0$ as $t \rightarrow \infty$. Then, from relation (18) $C_{i} A(t) \epsilon^{i}(t) \rightarrow 0$ as $t \rightarrow \infty$.

In the following, we have to prove that $\epsilon^{i}(t) \rightarrow 0$ as $t \rightarrow \infty$ by using again the Babalat's lemma. To this end, we compute the time derivatives of the function $\varphi(t)=C_{i} A(t) \epsilon^{i}(t)$. A simple calculation gives

$$
\dot{\varphi}(t)=\left(C_{i} \dot{A}-\gamma_{i} C_{i} A A^{T} C_{i}^{T} C_{i} A\right) \epsilon^{i}
$$

It is easy to show that if Assumption (1) holds and if $\epsilon^{i}(t)$ is bounded, then $\ddot{\varphi}(t)$ is bounded which implies that $\dot{\varphi}(t)$ is uniformly continuous. Since $\varphi(t) \rightarrow 0$ and $\dot{\varphi}(t)$ is uniformly continuous it follows from Barbalat's lemma that $\dot{\varphi}(t)=\left(C_{i} \dot{A}-\gamma C_{i} A A^{T} C_{i}^{T} C_{i} A\right) \epsilon^{i} \rightarrow 0$ as $t \rightarrow \infty$. Now since $\dot{\varphi}(t) \rightarrow 0$ and $C_{i} A \epsilon^{i} \rightarrow 0$ then $C_{i} \dot{A} \epsilon^{i} \rightarrow 0$. 
Now, without loss of generality set $i=1$ then the two conditions $C_{i} A \epsilon^{i} \rightarrow 0$ and $C_{i} \dot{A} \epsilon^{i} \rightarrow 0$ can be written as

$$
\left\{\begin{array}{lll}
r(t) \epsilon_{2}^{1}(t)-q(t) \epsilon_{3}^{1}(t) & \rightarrow & 0 \\
-r(t) \epsilon_{1}^{1}(t)+p(t) \epsilon_{3}^{1}(t) & \rightarrow & 0
\end{array}\right.
$$

and

$$
\left\{\begin{array}{lll}
\dot{r}(t) \epsilon_{2}^{1}(t)-\dot{q}(t) \epsilon_{3}^{1}(t) & \rightarrow & 0 \\
-\dot{r}(t) \epsilon_{1}^{1}(t)+\dot{p}(t) \epsilon_{3}^{1}(t) & \rightarrow & 0
\end{array}\right.
$$

where $\epsilon_{j}^{i}$ is the $j^{\text {th }}$ component of the observation error $\epsilon^{i}$. Using the first relations from (20) and (21) it is easy to see that $(q(t) \dot{r}(t)-\dot{q}(t) r(t)) \epsilon_{3}^{1}(t) \rightarrow 0$. Suppose that the function $q(t) \dot{r}(t)-\dot{q}(t) r(t)$ (the first component of vector $\vec{\omega} \wedge \dot{\vec{\omega}})$ does not go to zero at the inifinity, then $\epsilon_{3}^{1} \rightarrow 0$. If $r(t) \rightarrow 0$, then $\dot{r}(t) \rightarrow 0$ as $\dot{r}(t)$ is uniformly continuous which contradicts assumption (2) since $q(t) \dot{r}(t)-\dot{q}(t) r(t)$ does not go to zero at the infinity. Notice that in the last expression $q$ and $\dot{q}$ are bounded. Using $\epsilon_{3}^{1}(t) \rightarrow 0$, from relation (20), it is obvious that $\epsilon_{1}^{1}(t) \rightarrow 0$ and $\epsilon_{2}^{1}(t) \rightarrow 0$. The same reasoning can be used with the second component of the vector $\vec{\omega} \wedge \dot{\vec{\omega}}$ which proves the asymptotical stability of the observation error. The same approach can be easily applied for the SFORG $\mathcal{O}_{2}$ and $\mathcal{O}_{3}$. Now using relation (16), the residual and the sensor fault observation error can be written as

$$
\begin{aligned}
r_{i}(t) & =-\beta_{i} \gamma_{i} C_{i} A^{T}(t) C_{i}^{T} C_{i} A(t) \epsilon^{i}(t) \\
\epsilon_{f}^{i}(t) & =\gamma_{i} \bar{C}_{i} A^{T}(t) C_{i}^{T} C_{i} A(t) \epsilon^{i}(t)
\end{aligned}
$$

Thanks to relations (22) and (23), it is easy to see that if $\bar{f}^{i}(t) \equiv 0$ then $\epsilon_{f}^{i} \rightarrow 0$ and $r_{i} \rightarrow 0$ since $\epsilon^{i} \rightarrow 0$. This ends the first step of the proof.

Now consider the case $\bar{f}^{i}(t) \neq 0$ or equivalently at least one of $f_{j} \neq 0$ for $j \neq i$. First, one can see that $\epsilon^{i}(t) \nrightarrow 0$ and the residual can be written as

$$
r_{i}(t)=S_{i}(t) \epsilon^{i}(t)+T_{i}(t) \bar{f}^{i}(t)
$$

with $S_{i}=-\gamma_{i} \beta_{i} C_{i} A^{T}(t) C_{i}^{T} C_{i} A(t)$ and $T_{i}=-\beta_{i} C_{i}\left(L_{i}+\gamma_{i} A^{T}(t) C_{i}^{T}\right)+\beta_{i} I=-\beta_{i} \gamma_{i} C_{i} A^{T}(t) C_{i}^{T}$ which lead to $S_{1}=-\gamma_{1} \beta_{1} p\left[\begin{array}{ccc}-q & p & 0 \\ -r & 0 & p\end{array}\right], S_{2}=-\gamma_{2} \beta_{2} q\left[\begin{array}{ccc}q & -p & 0 \\ 0 & -r & q\end{array}\right], S_{3}=-\gamma_{3} \beta_{3} r\left[\begin{array}{ccc}r & 0 & -p \\ 0 & r & -q\end{array}\right], T_{1}=-\gamma_{1} \beta_{1}\left[\begin{array}{cc}0 & -p \\ p & 0\end{array}\right]$, $T_{2}=-\gamma_{2} \beta_{2}\left[\begin{array}{cc}0 & q \\ -q & 0\end{array}\right]$ and $T_{3}=-\gamma_{3} \beta_{3}\left[\begin{array}{cc}0 & -r \\ r & 0\end{array}\right]$. Assume that there exist $\left(\bar{f}_{p}^{i}(t) \neq 0, \epsilon_{p}^{i}(t)\right)$ such that $r_{i}(t)=S_{i}(t) \epsilon_{p}^{i}(t)+T_{i}(t) \bar{f}_{p}^{i}(t)=0$ then

$$
\bar{f}_{p}^{i}(t)=-T_{i}^{-1}(t) S_{i}(t) \epsilon_{p}^{i}(t)
$$

Inserting (25) in (16) yields

$$
\dot{\epsilon}_{p}^{i}(t)=A(t) \epsilon_{p}^{i}(t)
$$

By developing relation (25) for $i=1$ to 3 we can characterize all the non detectable sensor failures $f_{p k}$ which verify

$$
f_{p k}=\dot{\sigma}_{k}
$$

such that $\sigma$ is solution of $(26)$ and $\sigma_{k}$ is the $k^{\text {th }}$ component of $\sigma$. More precisely, if there exist $f_{j}(t) \neq 0$ for $j \neq i$ and $f_{j}(t) \neq \dot{\sigma}_{j}$ with $\sigma$ solution of $\dot{\sigma}(t)=A(t) \sigma(t)$ then $r_{i}(t) \neq 0$ and $r_{i}(t) \nrightarrow 0$. This ends the proof.

Remark 3. The possibility that a sensor fault is of the form $f_{p k}=\dot{\sigma}_{k}$ with $\sigma$ solution of $\dot{\sigma}(t)=A(t) \sigma(t)$ is rather not very probable. We can thus detect, isolate and identify the majority of constant and time varying sensor fault.

Remark 4. In the case of a single sensor failure $f_{i}$, the observer $\left(\mathcal{O}_{i}\right)$ gives an estimation of the state and the fault and the residual $r_{i}$ vanishes to 0 . In addition the other residuals are different from zero.

Remark 5. Our approach can detect, isolate, identify and estimate the fault in the case of single sensor failure. In case where two or three sensors are failing, we can only detect the presence of failure because all the residues are different from and do not converge to zero. 


\section{$5 \quad$ Numerical simulation}

In this section, we applied our approach to design a bank of state and sensor fault observer and residual generator for an UAV. The simulations are performed with the following parameters ([9]) $\mathrm{m}=2.5 \mathrm{~kg}$, $I_{x x}=22493110^{-7} \mathrm{~kg} \cdot \mathrm{m}^{2}, I_{y y}=22261110^{-7} \mathrm{~kg} \cdot \mathrm{m}^{2}$ and $I_{z z}=32513010^{-7} \mathrm{~kg} \cdot \mathrm{m}^{2}$.

We assume that the sensor fault signals are $f_{1}(t) \equiv 0, f_{2}(t) \equiv 0$ and $f_{3}(t)=\mathcal{H}(t-2.5)(0.6+\sin (20 \pi t+1))$ where $\mathcal{H}($.$) is the Heaviside function.$

The following figures represent the state observation errors (fig. 3 to fig. 5) for the SFORG $\mathcal{O}_{2}$ and $\mathcal{O}_{3}$, the residual signals $r_{2}(t)$ and $r_{3}(t)$ (fig. 6 and fig. 7 ) and the sensor fault signal $f_{3}(t)$ and its estimation $\hat{f}_{3}(t)$ (fig. 8).

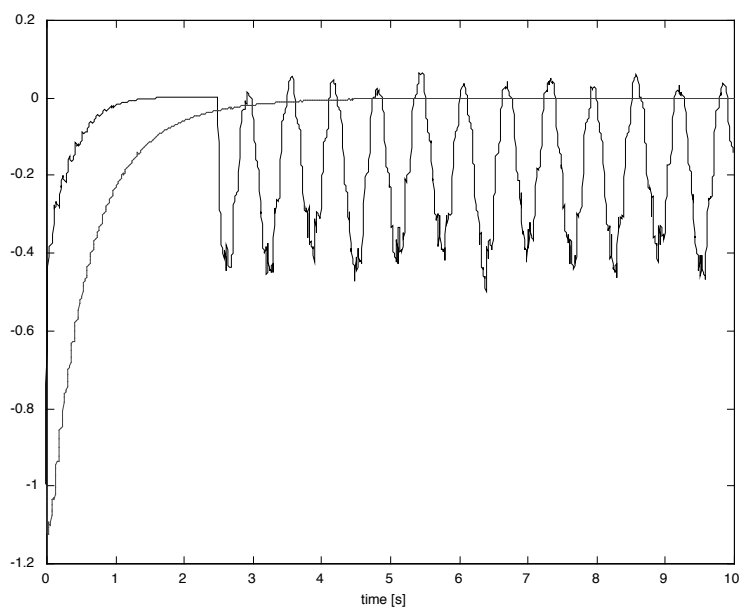

Figure 3: Observation errors $\varepsilon_{1}^{2}(t)$ and $\varepsilon_{1}^{3}(t)$.

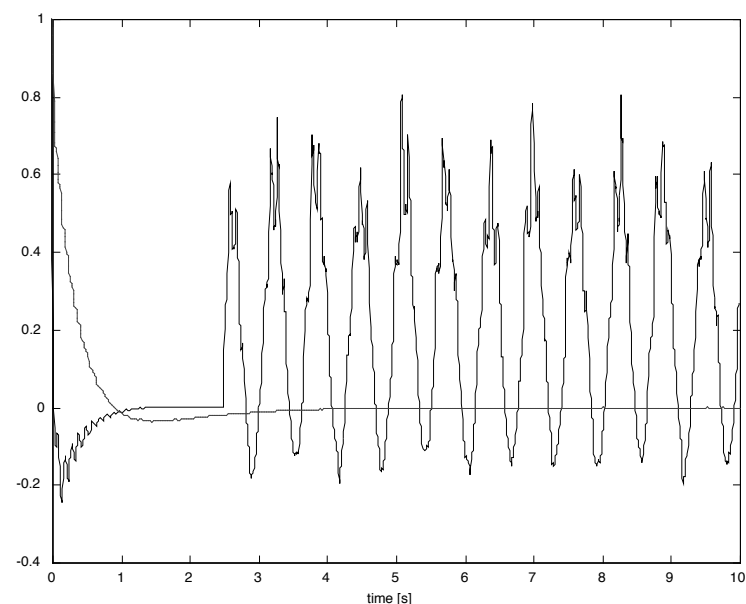

Figure 4: Observation errors $\varepsilon_{2}^{2}(t)$ and $\varepsilon_{2}^{3}(t)$. 


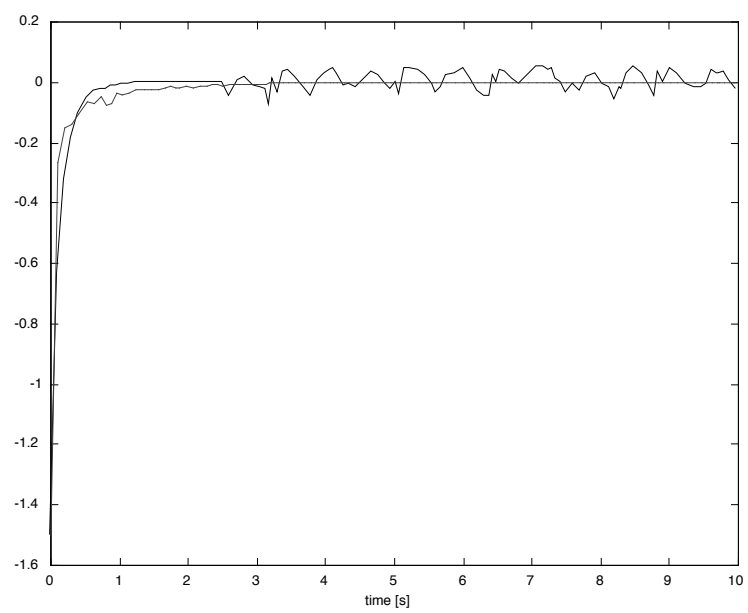

Figure 5: Observation errors $\varepsilon_{3}^{2}(t)$ and $\varepsilon_{3}^{3}(t)$.

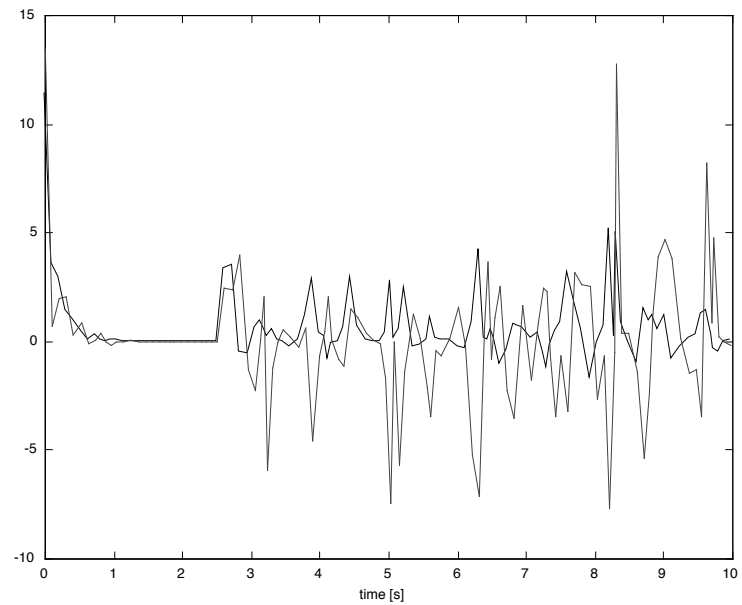

Figure 6: Residual signals $r_{2}(t)$.

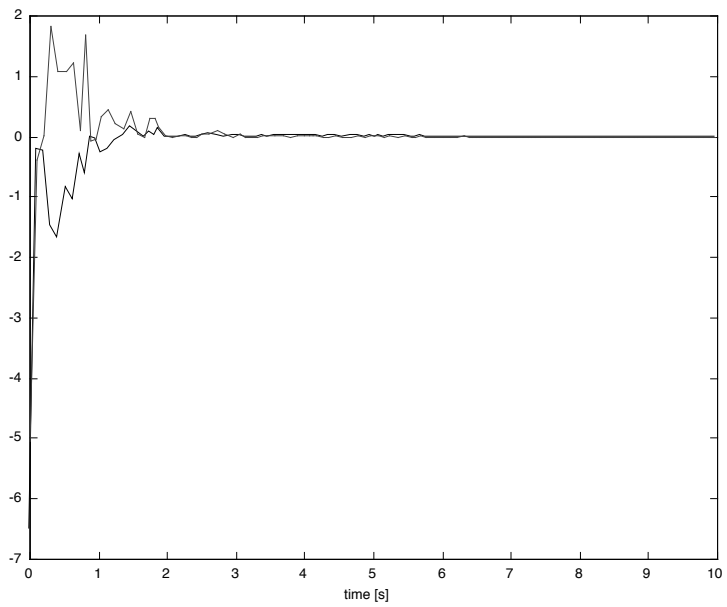

Figure 7: Residual signals $r_{3}(t)$. 


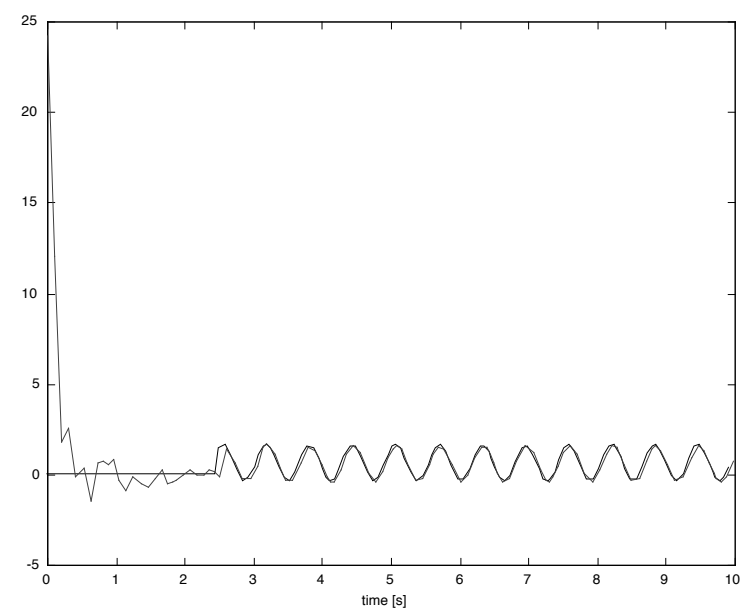

Figure 8: Sensor fault signal $f_{3}(t)$ and its estimation $\hat{f}_{3}(t)$.

One can see that all the observation errors (fig. 3 to fig. 5) of the SFORG $\mathcal{O}_{3}$ (solid lines) are asymptotically stable contrary to those given by the SFORG $\mathcal{O}_{2}$ (dashed lines). It can be noticed that the residual signals $r_{3}(t)$ (fig. 7) are asymptotically stable while $r_{2}(t)$ (fig. 6) are sensitive to the sensor fault signal $f_{3}(t)$. Finally, it is shown in fig. 8 that the reconstruction of the sensor fault signal is satisfactory.

\section{Conclusion}

In this note we addressed the problem of speed estimation of UAV under a sensor diagnosis procedure. This is based on a bank of reduced order estimators to simultaneously isolate/ estimate faults as well as the speed of the UAV from accelerometers. The main contributions concern the stability analysis of the observation errors and the fact of highlighting all trajectories of faults which can be detected. Simulation results show effectiveness of the proposed approach.

\section{References}

[1] D. B. Lee, T. C. Burg, B. Xian and D. M. Dawson, "Output feedback tracking control of an underactuated quad-rotor UAV", American Control Conference, New York, 2007.

[2] T. Madani and A. Benallegue, "Sliding mode observer and backstepping control for a quadrotor unmanned aerial vehicles", American Control Conference, New York, 2007.

[3] A. Benallegue, A. Mokhtari and L. Fridman, "High-order sliding-mode observer for a quadrotor UAV", Int. J. of Robust and Nonlinear Control, 2008.

[4] F. LeBras, T. Hamel and R. Mahony, "Nonlinear observer-based visual control of a VTOL UAV", European Control Conference, Kos, Greece, 2007.

[5] R. Sharma and M. Aldeen, "Fault and unknown input reconstruction in VTOL aircraft system using sliding mode observer", European Control Conference, Kos, Greece, 2007.

[6] F. Bateman, H. Noura and M. Ouladsine, "An actuator fault detection, isolation and estimation system for an UAV using input observers", European Control Conference, Kos, Greece, 2007.

[7] N. Slegers and M. Costello, "Variable structure observer for control bias on unmanned air vehicles", J. of Guidance, Cont., and Dynamics, vol. 30, pp 281-286, 2007. 
[8] C. G. Prevost, A. Desbiens and E. Gagnon, "Extended Kalman filter for state estimation and trajectory prediction of a moving object detected by an unmanned aerial vehicle", American Control Conference, New York, 2007.

[9] K. Benzemrane, G. L. Santosuosso and G. Damm, "Unmanned aerial vehicle speed estimation via nonlinear adaptive observers", American Control Conference, New York, 2007.

[10] L. Besnard, Y.B. Shtessel and B. Landrum, "Control of a quadrotor vehicle using sliding mode disturbance observer", American Control Conference, New York, 2007.

[11] D. Blake and M. Brown, "Simultaneous, multiplicative actuator and sensor fault estimation", European Control Conference, Kos, Greece, 2007.

[12] M. Boutayeb, E. Richard, H. Rafaralahy, H. Souley Ali and G. Zaloylo, "A simple time-varying observer for speed estimation of UAV", IFAC World Congress, Seoul, Korea, 2008.

[13] P. Castillo, R. Lozano and A. Dzul, Modelling and control of mini-flying machines, Springer-Verlag, 2005.

[14] J. C. Deckert, M. N. Desai, J. J. Deyst and A. S. Willsky, "F-8 DFBW sensor failure identification using analytic redundancy", IEEE Trans. Aut. Cont., Vol. 22, 5, 1977.

[15] D. Lara Alabazares, Modélisation et commande robuste des drones miniatures : conception de l'architecture embarquée, PhD thesis, UTC, Compiegne, France, 2007.

[16] A. Monteriu, P. Asthana, K. Valavanis and S. Longhi, "Residual generation approaches in navigation sensors fault detection applications", European Control Conference, Kos, Greece, 2007.

[17] H. Nijmeijer, A. J. van der Schaft, nonlinear dynamical control systems, Springer-Verlag, 1990.

[18] N. P. Piercy, "Sensor failure estimators for detection filters", IEEE Trans. Aut. Cont., Vol. 37, 10, 1992.

[19] H. Rafaralahy, M. Zasadzinski and M. Boutayeb, "Discussion on sensor gain fault diagnosis for a class of nonlinear systems", European Journal of Control, Vol. 12, 5, 2006.

[20] H. Rafaralahy, M. Zasadzinski, M. Boutayeb and H. Souley Ali, "Sensor bias estimation for bilinear systems", Conference on Systems and Control, Marrakesh, Morocco, 2007.

[21] Y. Wang and D.H. Zhou, "Sensor gain fault diagnosis for a class of nonlinear systems", European Journal of Control, Vol. 12, 5, pp. 523-535, 2006. 